\title{
Safety, infectivity and immunogenicity of a genetically attenuated blood-stage malaria vaccine
}

Rebecca Webster ${ }^{1}$, Silvana Sekuloski ${ }^{1,2}$, Anand Odedra ${ }^{1,3}$, Stephen Woolley ${ }^{1,3,4}$, Helen Jennings ${ }^{1}$, Fiona Amante ${ }^{1}$, Katharine R. Trenholme ${ }^{1,5}$, Julie Healer ${ }^{6}$, Alan F. Cowman ${ }^{6,7}$, Emily M. Eriksson ${ }^{6,8}$, Priyanka Sathe ${ }^{9}$, Jocelyn Penington ${ }^{6}$, Adam J. Blanch ${ }^{10,11}$, Matthew W. A. Dixon ${ }^{10,11}$, Leann Tilley ${ }^{10,11}$, Michael F. Duffy ${ }^{7,10,12,13}$ Alister Craig ${ }^{3}$, Janet Storm ${ }^{3}$, Jo-Anne Chan ${ }^{14}$, Krystal Evans ${ }^{6,15}$, Anthony T. Papenfuss ${ }^{6,8}$, Louis Schofield ${ }^{6,16}$, Paul Griffin ${ }^{1,5,17}$, Bridget E. Barber ${ }^{1}$, Dean Andrew ${ }^{1}$, Michelle J. Boyle ${ }^{1}$, Fabian de Labastida Rivera', Christian Engwerda ${ }^{1}$ and James S. McCarthy ${ }^{1,12^{*}}$

\section{Abstract}

Background: There is a clear need for novel approaches to malaria vaccine development. We aimed to develop a genetically attenuated blood-stage vaccine and test its safety, infectivity, and immunogenicity in healthy volunteers. Our approach was to target the gene encoding the knob-associated histidine-rich protein (KAHRP), which is responsible for the assembly of knob structures at the infected erythrocyte surface. Knobs are required for correct display of the polymorphic adhesion ligand $P$. falciparum erythrocyte membrane protein 1 (PfEMP1), a key virulence determinant encoded by a repertoire of var genes.

Methods: The gene encoding KAHRP was deleted from P. falciparum 3D7 and a master cell bank was produced in accordance with Good Manufacturing Practice. Eight malaria naïve males were intravenously inoculated (day 0) with 1800 ( 2 subjects), $1.8 \times 10^{5}$ (2 subjects), or $3 \times 10^{6}$ viable parasites (4 subjects). Parasitemia was measured using qPCR; immunogenicity was determined using standard assays. Parasites were rescued into culture for in vitro analyses (genome sequencing, cytoadhesion assays, scanning electron microscopy, var gene expression).

Results: None of the subjects who were administered with 1800 or $1.8 \times 10^{5}$ parasites developed parasitemia; $3 / 4$ subjects administered $3 \times 10^{6}$ parasites developed significant parasitemia, first detected on days 13,18 , and 22 . One of these three subjects developed symptoms of malaria simultaneously with influenza B (day 17; 14,022 parasites/ $\mathrm{mL})$; one subject developed mild symptoms on day $28(19,956$ parasites $/ \mathrm{mL}) ;$ and one subject remained asymptomatic up to day 35 (5046 parasites $/ \mathrm{mL}$ ). Parasitemia rapidly cleared with artemether/lumefantrine. Parasitemia induced a parasite-specific antibody and cell-mediated immune response. Parasites cultured ex vivo exhibited genotypic and phenotypic properties similar to inoculated parasites, although the var gene expression profile changed during growth in vivo.

\footnotetext{
* Correspondence: james.mccarthy@unimelb.edu.au

${ }^{1}$ QIMR Berghofer Medical Research Institute, Brisbane, Australia

${ }^{12}$ The Peter Doherty Institute for Infection and Immunity, Melbourne, Australia

Full list of author information is available at the end of the article
}

C The Author(s). 2021 Open Access This article is licensed under a Creative Commons Attribution 4.0 International License, which permits use, sharing, adaptation, distribution and reproduction in any medium or format, as long as you give appropriate credit to the original author(s) and the source, provide a link to the Creative Commons licence, and indicate if changes were made. The images or other third party material in this article are included in the article's Creative Commons licence, unless indicated otherwise in a credit line to the material. If material is not included in the article's Creative Commons licence and your intended use is not permitted by statutory regulation or exceeds the permitted use, you will need to obtain permission directly from the copyright holder. To view a copy of this licence, visit http://creativecommons.org/licenses/by/4.0/. The Creative Commons Public Domain Dedication waiver (http://creativecommons.org/publicdomain/zero/1.0/) applies to the data made available in this article, unless otherwise stated in a credit line to the data. 
Conclusions: This study represents the first clinical investigation of a genetically attenuated blood-stage human malaria vaccine. A P. falciparum 3D7 kahrp- strain was tested in vivo and found to be immunogenic but can lead to patent parasitemia at high doses.

Trial registration: Australian New Zealand Clinical Trials Registry (number: ACTRN12617000824369; date: 06 June 2017).

Keywords: Malaria, Vaccine, Plasmodium falciparum, Genetically attenuated, KAHRP, PfEMP1

\section{Background}

There is currently a strong commitment to eradicate malaria in the 21 st century. This ambitious goal will be facilitated by the development of an effective vaccine. The past 20 years have seen over 100 clinical trials of malaria vaccine candidates, with the vast majority testing sub-unit vaccines [1]. To date, only one vaccine (RTS,S/ AS01) has demonstrated protective efficacy against clinical malaria in a phase 3 trial, although protection is only partial [2]. RTS,S/AS01 has been approved by the European Medicines Agency, and a pilot implementation program coordinated by the World Health Organization is underway in Africa [3]. Given the overall slow progress of current vaccine strategies, there is a clear need for novel approaches.

Live, whole cell vaccines offer several potential advantages over subunit vaccines against a range of pathogens including malaria parasites. These include exposure to a greater range of antigens that are delivered to the correct anatomical compartments for immunological priming, as well as providing the correct pattern recognition signals to the immune system, which are specific for the pathogen class [4]. Various whole sporozoite vaccines that target the pre-erythrocytic stage of malaria parasite development have demonstrated promising results in preclinical studies, human challenge studies, and in clinical trials in malaria-endemic areas [1]. Strategies for sporozoite attenuation have included the use of radiation [5] or targeted gene deletion [6-8] to produce genetically attenuated parasites (GAP). Naturally acquired immunity to malaria is complex, with evidence suggesting that repeated exposure to blood-stage infection is an important component and is associated with an antibody response against merozoites and infected erythrocytes [9]. However, in the modern era, only a single live whole parasite blood-stage vaccine candidate has been tested in humans, in a pilot study undertaken using chemically attenuated parasites [10]. We aimed to develop a bloodstage GAP malaria vaccine and characterize its safety, infectivity, and immunogenicity in a phase 1 clinical trial.

P. falciparum erythrocyte membrane protein 1 (PfEMP1) is a polymorphic adhesion ligand displayed on knob-like structures on the surface of P. falciparum-infected erythrocytes, with specific types binding a variety of host receptors present on the vascular endothelium [11]. This arrangement facilitates attachment and sequestration of the infected erythrocyte in the microvasculature, thereby protecting parasites from clearance by the spleen [12]. Further, parasite sequestration is a critical determinant of malaria pathogenesis as it leads to the accumulation of infected erythrocytes in vital organs [13]. PfEMP1 is also a target of protective immunity [14] and has been shown to have an immunomodulatory effect [15]. PfEMP1 is a variable surface antigen, encoded by a repertoire of around $60 \mathrm{var}$ genes per genome [16]. Thus, creation of a genetically modified parasite lacking PfEMP1 would require a targeted genetic modification that would prevent transcription of any var gene allele, an approach that may be technically challenging. Further, if such a result were achieved it would result in no expression of PfEMP1, a protein which has been proposed to be the target of strain-specific immunity [14].

The in vivo virulence and immunogenicity of a knobless $P$. falciparum clone selected under in vitro culture was investigated in Aotus monkeys by Langreth and Peterson [17]. After multiple inoculations with the knobless parasites, non-splenectomized animals either did not develop patent parasitemia, or exhibited very low parasitemia, with infections self-resolving. In contrast, splenectomized animals developed significant infections when inoculated with knobless parasites. This study indicated the importance of the knobs on the surface of infected erythrocytes in enabling parasites to sequester to avoid splenic clearance. When monkeys with intact spleens were exposed to knobless parasites they developed a humoral immune response and exhibited partial protection when subsequently challenged with knobpositive parasites. Together, these data suggest a knobminus live malaria vaccine represents an attractive approach.

Our approach to develop a blood-stage GAP vaccine was to target the gene encoding the knob-associated histidine-rich protein (KAHRP), which is responsible for the assembly of knob structures at the infected erythrocyte surface. In the absence of KAHRP, $P$. falciparum-infected erythrocytes lack knob-structures and cannot correctly display PfEMP1 [18]. Building on the early studies in Aotus monkeys, we hypothesized that the loss 
of KAHRP would result in a significant loss of fitness in vivo due to enhanced parasite clearance accompanied by priming of the immune system from lack of parasite sequestration and increased passage to the spleen. The production of the blood-stage GAP vaccine under Good Manufacturing Practice (GMP) by targeted deletion of the gene encoding KAHRP has been described previously [19]. Here we report the results of a clinical trial to characterize the safety, infectivity, and immunogenicity of the P. falciparum kahrp- blood-stage GAP vaccine in healthy volunteers.

\section{Methods}

\section{Study design and participants}

This phase 1 study was planned to be composed of two parts. The first part, performed as planned, was an openlabel, dose-escalation study to determine the safety, infectivity, and immunogenicity of single doses of the GAP vaccine administered intravenously. The second part was planned to provide preliminary information on the efficacy of the GAP vaccine by determining the protective immune response of GAP vaccination to subsequent challenge with wild-type blood-stage P. falciparum. However, it was decided not to proceed with the second part due to the lack of complete attenuation of the GAP vaccine, and alloimmunization events, observed in the first part (see the "Results" section). The study design and methodology for the second part of the study are not described here.

Healthy malaria naïve males aged 18 to 55 years were eligible for inclusion in the study (for full eligibility criteria see Additional File 1: Text S1). The study was conducted at Q-Pharm (Brisbane, Australia) and was registered on the Australian and New Zealand Clinical Trials Registry (Trial ID: ACTRN12617000824369; date registered: 06 June 2017).

\section{GAP vaccine}

The manufacture of the GAP vaccine has been described previously [19]. Briefly, genetically attenuated bloodstage malaria parasites were produced by targeted deletion of kahrp from P. falciparum strain 3D7, and parasites were cultured in a wave bioreactor under GMPcompliant conditions. The cryopreserved GAP master cell bank was tested for sterility, absence of viral contaminants and endotoxins, and parasite identity, viability, and antimalarial drug sensitivity. The GAP master cell bank has a parasitemia of $6.3 \%$ with $96 \%$ of parasites at ring stage. The GAP vaccine inoculum used for each subject in this study was prepared aseptically from a single vial of the GAP master cell bank by thawing, washing, and suspending the appropriate number of infected erythrocytes in $0.9 \%$ sodium chloride for injection in a final volume of $2 \mathrm{~mL}$. Syringes containing the inocula were stored at a controlled temperature for a maximum of $2 \mathrm{~h}$ before administration.

\section{Procedures}

The study was conducted in four consecutive dose cohorts of two subjects each. Sample size was not based on formal statistical calculations but was considered appropriate to achieve the study objectives. Following a screening period of up to 28 days, subjects were inoculated intravenously with the GAP vaccine (day 0). A sentinel dosing strategy was used for the first three cohorts whereby the first subject in each cohort was inoculated with the GAP vaccine at least $24 \mathrm{~h}$ before the second subject. Subjects in cohort 4 were inoculated with the GAP vaccine concurrently since no significant safety signals had been observed in the preceding cohorts.

Parasitemia was monitored throughout the study by collecting blood samples and performing quantitative PCR (qPCR) targeting the gene encoding $P$. falciparum $18 \mathrm{~S}$ rRNA [20]. Subjects were to receive a standard curative course of artemether/lumefantrine treatment if parasitemia reached $\geq 5000$ parasites $/ \mathrm{mL}$ ( $\geq 200,000$ parasites/ $\mathrm{mL}$ for cohort 4) or if clinical symptoms of malaria were observed (clinical symptom score $>6$ ), or on day $28 \pm 3$ (day $60 \pm 3$ for cohort 4 ) if the preceding treatment criteria had not been reached earlier. The end of the study visit occurred on day 90 for safety and immunogenicity assessments.

The GAP vaccine dose administered to cohort 1 was 1800 viable parasites which is the dose of $P$. falciparum 3D7 wild-type parasites routinely used in previous controlled human malaria infection (CHMI) studies using the induced blood-stage malaria (IBSM) model [21-23]. A formal Safety Review Team meeting was conducted between cohorts to assess the safety of the GAP vaccine and decide whether to dose escalate and proceed with the next cohort. The planned dose escalation was $1.8 \times$ $10^{5}$ viable parasites for cohort 2 (100-fold increase from cohort 1) and $3 \times 10^{6}$ viable parasites for cohort 3 (a dose of parasites equivalent to that released from the liver after a five mosquito bite CHMI study [24]). Based on the results observed in cohort 3 (with both subjects developing parasitemia but remaining asymptomatic), the study protocol was amended to include a fourth cohort administered the same GAP vaccine dose as cohort $3\left(3 \times 10^{6}\right.$ viable parasites), but with the antimalarial treatment criteria amended with a longer follow-up to allow for better characterization of parasite infectivity. A planned protocol deviation was performed for cohort 3 due to the unexpected results observed; subject 6 was administered antimalarial treatment on day 35 when parasitemia reached 5000 parasites $/ \mathrm{mL}$ instead of the protocol stipulated day 28. This was considered reasonable since the subject was asymptomatic and consented 
to the additional period of follow-up. The QIMR Berghofer Medical Research Institute Human Research Ethics Committee was notified of this decision.

\section{Safety assessments}

Safety assessments were performed throughout the study and included adverse event (AE) recording, physical examinations, vital signs monitoring, electrocardiographs (ECGs), clinical laboratory evaluation (hematology, biochemistry, and urinalysis), and malaria clinical score recording [25]. The severity of AEs was graded according to a set of criteria developed for guidance of commonly reported symptoms, signs, and abnormal laboratory findings in malaria challenge studies $($ mild $=$ grade 1 ; moderate $=$ grade 2 ; severe $=$ grade 3 ). These were adapted from the Common Terminology Criteria for Adverse Events v3.0 [26].

\section{Immunogenicity assessments}

The development of an antibody response to the GAP vaccine was determined by collecting blood samples and using standard ELISA methods to measure IgG and IgM antibodies towards P. falciparum merozoite surface protein 2 (MSP2) and to whole parasite lysate (Additional File 2: Text S1) [27, 28]. The development of a cell-mediated immune response to the GAP vaccine (cohorts 1-3 only) was determined by collecting blood samples and using flow cytometry to identify $\mathrm{T}$ cell subsets (Additional File 2: Text S2, Table S1, and Figure S1) [29, 30].

\section{In vitro analysis of parasites rescued into culture}

A series of in vitro analyses were performed on parasites isolated from the blood of subjects at maximum parasitemia prior to antimalarial treatment. Parasites were cultured directly from blood samples to reach a target parasitemia of $2-8 \%$ with $>50 \%$ ring stage parasites before cryopreservation. The properties of the isolated parasites were compared to parasites inoculated on day 0 . Genotyping of the parasite genetic material was performed using standard molecular biology techniques including Sanger sequencing and whole genome sequencing (Additional File 3: Text S1) [31-36]. The cytoadherence phenotype of the isolates was determined using both static and flow adhesion assays (Additional File 3: Text S2) [37, 38]. Scanning electron microscopy was performed to characterize the knob status of parasite-infected erythrocytes (Additional File 3: Text S3) [39]. The var gene expression profile of parasites was determined using diagnostic RT-PCR (Additional File 3: Text S4 and Table S1) [40-43].

\section{Results}

Subjects

The study was conducted from 6 July 2017 to 11 September 2019. A total of 8 subjects were enrolled over four dose cohorts (Fig. 1). Subjects were healthy malarianaïve males between the ages of 18 and 48 years; five subjects self-selected their race as White, two as Latin Hispanic, and one as Australian Aboriginal (Table 1). All subjects completed the study and were included in the analysis of study endpoints.

\section{GAP vaccine infectivity}

Parasitemia was not detected in subjects in cohort 1 (administered 1800 viable parasites) or cohort 2 (administered $1.8 \times 10^{5}$ viable parasites) at any time-point during the study (Fig. 2). Parasitemia was detected in all four subjects in cohorts 3 and 4 (administered $3 \times 10^{6}$ parasites), although parasitemia was only detected very briefly and at a very low level (around the limit of detection) in one of these subjects (subject 8, day 1 and day 38 , maximum 3 parasites $/ \mathrm{mL}$ ). The other three subjects developed significant parasitemia, the progression of which was highly variable between subjects. Antimalarial treatment with artemether/lumefantrine was initiated on day 17 , day 28 , and day 35 for these subjects at a parasitemia of 14,022 parasites/mL, 19,956 parasites/mL, and 5151 parasites $/ \mathrm{mL}$, respectively (Fig. 2, arrows). Parasitemia was rapidly cleared following artemether/lumefantrine treatment and no recrudescence was observed up to day 90 (Fig. 2).

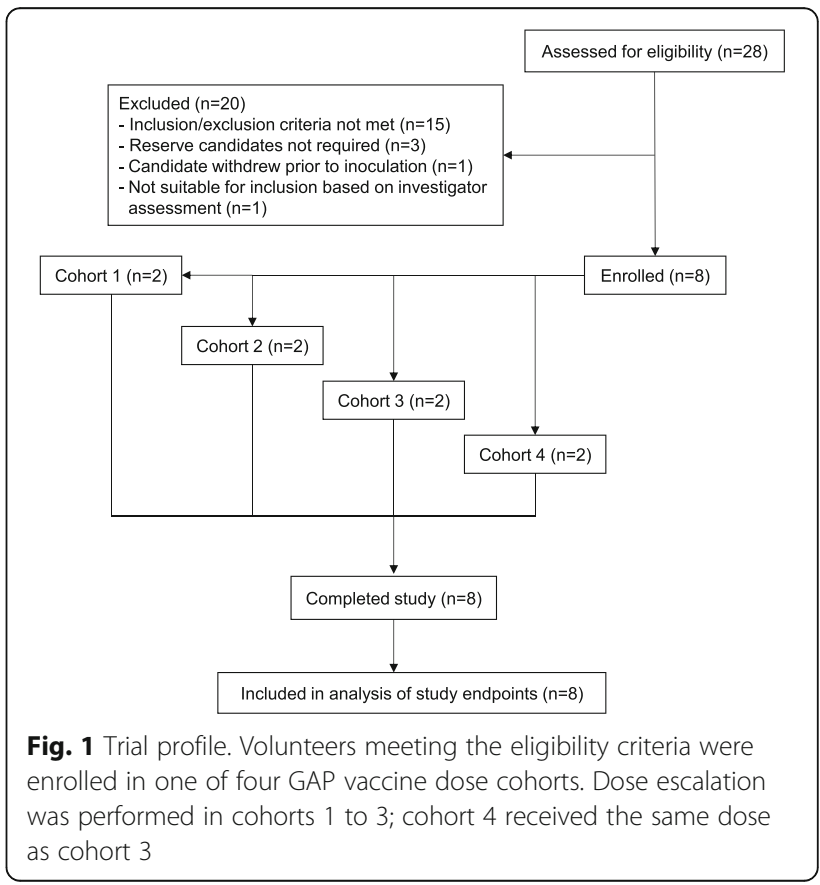


Table 1 Demographic profile of subjects

\begin{tabular}{|c|c|c|c|c|c|c|c|c|}
\hline & \multicolumn{2}{|l|}{ Cohort 1} & \multicolumn{2}{|l|}{ Cohort 2} & \multicolumn{2}{|l|}{ Cohort 3} & \multicolumn{2}{|l|}{ Cohort 4} \\
\hline & Subject 1 & Subject 2 & Subject 3 & Subject 4 & Subject 5 & Subject 6 & Subject 7 & Subject 8 \\
\hline GAP vaccine dose (viable parasites) & 1800 & & $1.8 \times 10^{5}$ & & $3 \times 10^{6}$ & & $3 \times 10^{6}$ & \\
\hline Age (years) & 40 & 48 & 26 & 19 & 32 & 18 & 29 & 20 \\
\hline Sex & Male & Male & Male & Male & Male & Male & Male & Male \\
\hline Race & Latin Hispanic & Latin Hispanic & White & Australian Aboriginal & White & White & White & White \\
\hline Body mass index $\left(\mathrm{kg} / \mathrm{m}^{2}\right)$ & 23.0 & 27.3 & 18.1 & 21.7 & 26.7 & 23.8 & 26.8 & 23.0 \\
\hline
\end{tabular}

$B M I$ body mass index

\section{Safety}

A total of 44 adverse events (AEs) were reported in this study, the majority of which were of mild severity (Table 2 and Additional File 1: Table S1). One AE met the protocol-defined criteria of a serious adverse event: a subject in cohort 4 experienced a joint dislocation on day 70 which required hospitalization and surgery. This event was not considered related to any study interventions. There were 15 AEs in 3 subjects (all administered the highest dose of $3 \times 10^{6}$ viable parasites) that were considered to be possibly related to the GAP vaccine. Twelve of

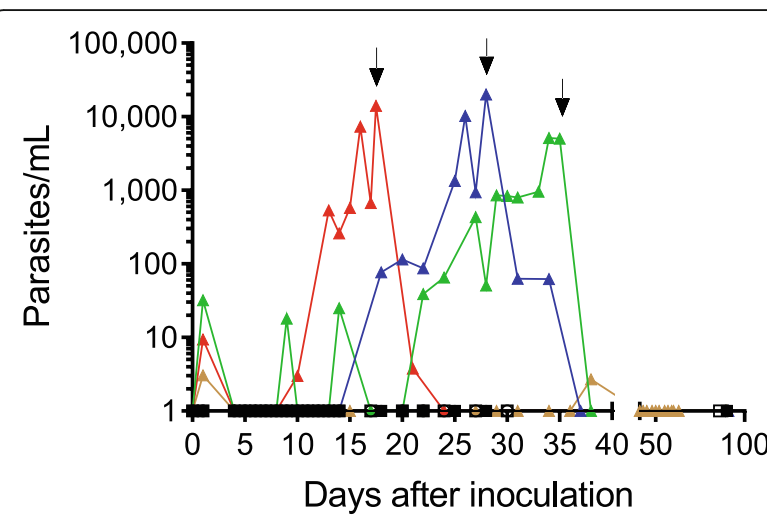

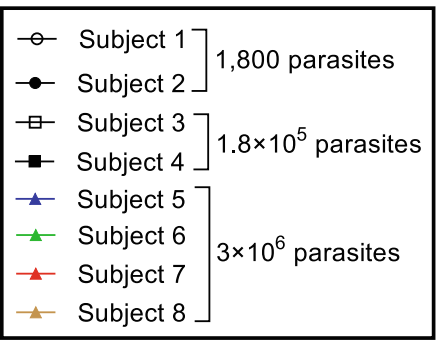

Fig. 2 Infectivity of the GAP vaccine. Parasitemia was monitored by collecting blood samples and performing qPCR targeting the gene encoding P. falciparum $18 \mathrm{~S}$ rRNA. Arrows indicate the commencement of treatment with artemether/lumefantrine for subjects who developed parasitemia. Time points at which parasitemia could not be detected were substituted with a value of 1 parasite/mL for the purpose of graphing on a logarithmic scale. Subject 7 was confirmed to be positive for influenza $B$ at the time of antimalarial treatment these AEs occurred in one subject (subject 7) and were mild symptoms/signs frequently associated with malaria (headache [ 2 events], myalgia, arthralgia [2 events], malaise, chills, pyrexia [ 2 events, maximum body temperature $38.9^{\circ} \mathrm{C}$ ], tachycardia [maximum heart rate 108 beats per minute], feeling hot, hyperhidrosis). However, this subject was confirmed by PCR to be positive for influenza B at the time of antimalarial treatment (which coincided with the onset of the majority of the signs/symptoms listed above); thus the symptoms and signs observed may not have been malaria-related. Another subject (subject 5) experienced mild fatigue ( $24 \mathrm{~h}$ duration) which was considered possibly related to the GAP vaccine.

Alloimmunization to minor erythrocyte antigens was observed in two subjects (subject 7 and subject 8 ) at the end of the study visit on day 90 , which was considered related to the GAP vaccine. Subject 7 developed low titer anti-C and anti-P1 antibodies, while subject 8 developed low titer anti-c antibodies. Retrospective testing was performed on blood samples collected on day 21 (subject 7) and day 31 (subject 8). Subject 7 was positive for anti-P1 on day 21 (there was an insufficient sample to test for anti-C at this time point); subject 8 was negative for anti-c on day 31. The blood group and phenotype of these subjects, as well as the donor red blood cells used to manufacture the GAP master cell bank, are presented in Additional File 1: Table S2. Alloimmunization was not observed in any of the other 6 subjects administered the GAP vaccine.

\section{Immunogenicity}

The three subjects who developed significant parasitemia following administration of $3 \times 10^{6}$ parasites (subject 5, subject 6 , and subject 7) also developed a malaria-specific antibody response. The antibody response to a key immunogenic blood-stage antigen (MSP2) was measured. The timing of the anti-MSP2 IgG response (Fig. 3A) and IgM response (Additional File 2: Figure S2) appeared to correlate with the development of parasitemia (Fig. 2). IgG and IgM antibody responses to whole parasite lysate were comparable to the MSP2 response in all subjects (Additional File 2: Figure S3). Furthermore, a malaria-specific 
Table 2 Summary of adverse events

\begin{tabular}{|c|c|c|c|c|c|}
\hline & $\begin{array}{l}\text { Cohort } 1 \\
(N=2)\end{array}$ & $\begin{array}{l}\text { Cohort } 2 \\
(N=2)\end{array}$ & $\begin{array}{l}\text { Cohort } 3 \\
(N=2)\end{array}$ & Cohort $4^{b}(N=2)$ & $\begin{array}{l}\text { Total } \\
(N=8)\end{array}$ \\
\hline \multicolumn{6}{|c|}{ Number of subjects with at least one adverse event [ $(\%)]$; number of adverse events } \\
\hline Any adverse event & $2(100 \%) ; 13$ & $1(50 \%) ; 3$ & $2(100 \%) ; 5$ & $2(100 \%) ; 23$ & $7(87.5 \%) ; 44$ \\
\hline Adverse event of moderate intensity & $1(50 \%) ; 3$ & $0(0 \%) ; 0$ & $1(50 \%) ; 1$ & $2(100 \%) ; 3$ & $4(50 \%) ; 7$ \\
\hline Adverse event of severe intensity & $0(0 \%) ; 0$ & $0(0 \%) ; 0$ & $0(0 \%) ; 0$ & $1(50 \%) ; 1^{a}$ & $1(12.5 \%) ; 1^{\mathrm{a}}$ \\
\hline Adverse event related to GAP vaccine & $0(0 \%) ; 0$ & $0(0 \%) ; 0$ & $1(50 \%) ; 1$ & $2(100 \%) ; 14$ & $3(37.5 \%) ; 15$ \\
\hline Serious adverse event & $0(0 \%) ; 0$ & 0 (0\%); 0 & $0(0 \%) ; 0$ & $1(50 \%) ; 1^{a}$ & $1(12.5 \%) ; 1^{\mathrm{a}}$ \\
\hline
\end{tabular}

${ }^{a}$ The severe adverse event (joint dislocation) also met the criteria for a serious adverse event since it required hospitalization and surgery. This event was not considered related to the GAP vaccine. ${ }^{b}$ One subject in cohort 4 (subject 7) was confirmed to be positive for influenza B at the time of antimalarial treatment

cell-mediated immune response to the GAP vaccine was identified in two of the three subjects (subject 5 and subject 6; not tested in subject 7) administered $3 \times 10^{6}$ parasites, including increased expression of activation markers PD-1 and ICOS on Th1-like Tfh cells (Fig. 3B) and in the expression of activation markers CD38 and HLA-DR on Th1 cells (Fig. 3C) on day 28. There was no change in the frequencies of $\mathrm{CD}^{+}{ }^{+} \mathrm{T}$ cells, Treg cells, Th1 cells, or Tfh cells in the peripheral blood of any of the subjects over the course of the study (Additional File 2: Figure S4).

\section{In vitro analyses of parasites rescued into culture}

In vitro analyses of parasites rescued into culture from blood samples collected at peak parasitemia were performed to determine if any genotypic or phenotypic changes occurred during growth in vivo. Whole genome sequencing revealed that there were no significant genetic changes between the inoculated parasites and the ex vivo isolates (Additional File 3: Text S5), confirming that parasites had not reverted to a wild-type genotype, and had not acquired new mutations that may

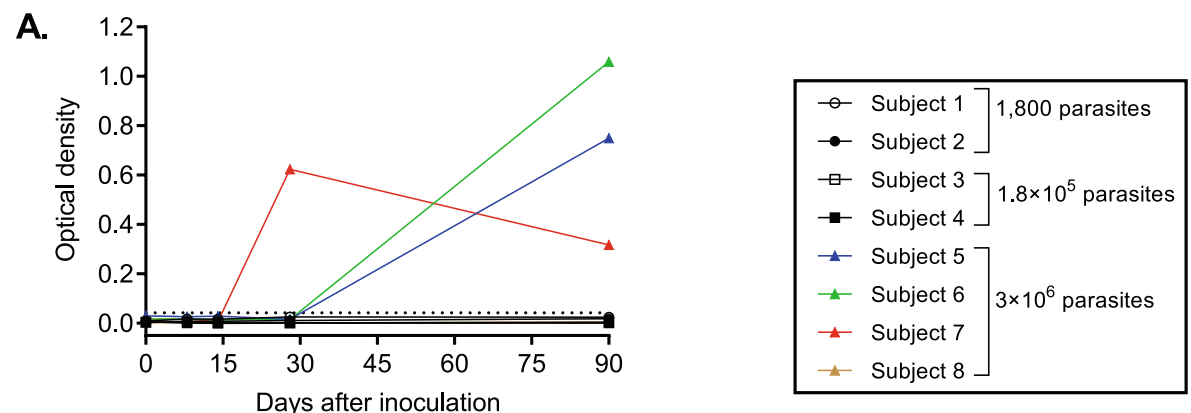

B.

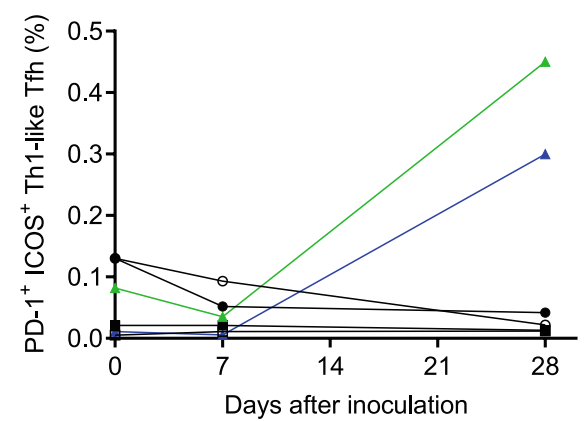

c.

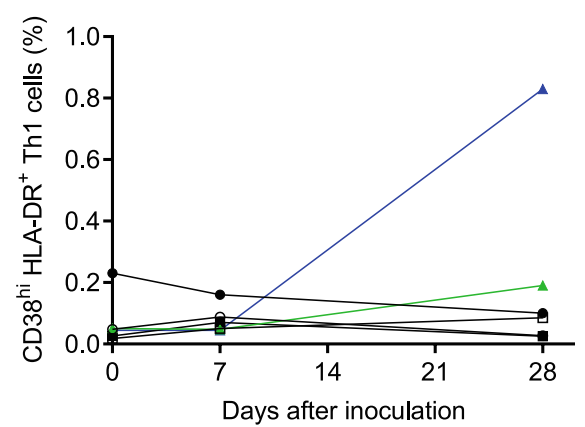

Fig. 3 Immunogenicity of the GAP vaccine. The parasite-specific antibody response was determined by collecting blood samples and measuring anti-MSP2 lgG over the course of the study in each subject (A). The cell-mediated immune response was determined by measuring the frequency of activated $\left(\mathrm{PD}-1^{+} \mathrm{ICOS}^{+}\right) \mathrm{CD}^{+}$Tfh cells (B) and activated $\left(\mathrm{CD} 38^{\text {hi }} \mathrm{HLA}-\mathrm{DR}^{+}\right)$Th1 cells $(\mathbf{C})$ in peripheral blood mononuclear cells over the course of the study in each subject. $C D 4^{+} T$ cell subsets were identified in B and $C$ as indicated in Additional File 2: Figure S1. The cellmediated immune response was not determined for cohort 4 (subject 7 and subject 8). 
compensate for KAHRP deficiency. The ex vivo parasiteinfected erythrocytes showed the same knob-minus appearance as infected erythrocytes inoculated on day 0 when examined using scanning electron microscopy (Fig. 4 and Additional File 3: Text S6) and retained an impaired cytoadherence phenotype in static and flow adhesion assays (Fig. 5 and Additional File 3: Text S7). Following growth in vivo, parasites switched away from primarily expressing a single group $\mathrm{C}$ var gene at baseline (inoculation), to elevated expression of most of the var repertoire in two subjects (subject 5 and subject 6) and a less broad but still fairly diverse pattern of expression in subject 7 (Fig. 6 and Additional File 3: Text S8). The most abundantly transcribed genes differed between the subjects and none had a published association with virulence (Additional File 3: Table S2) [44]. Wild-type $P$. falciparum 3D7 parasites from a CHMI study using the IBSM model [45] were analyzed as a control. The wildtype parasites used for inoculation of two subjects in that study expressed much of the var repertoire at elevated levels; the expressed repertoire following growth in vivo was largely unchanged in one subject but was less diverse in the other subject (Fig. 6).

\section{Discussion}

This study represents the first clinical investigation of a genetically attenuated blood-stage human malaria vaccine. We demonstrate that $P$. falciparum 3D7 parasites lacking KAHRP are highly attenuated in vivo compared to their wild-type counterparts, with no parasitemia detectable up to 28 days following inoculation of up to $1.8 \times 10^{5}$ viable parasites. Inoculation of healthy subjects with 1800-2800 wild-type $P$. falciparum 3D7 parasites in CHMI studies using the IBSM model consistently results in detectable parasitemia within 5 days, and parasitemia typically reaches $>5000$ parasites $/ \mathrm{mL} 8$ days after inoculation [21, 22, 25, 46, 47]. However, we found that P. falciparum kahrp- parasites were capable of generating a significant infection when administered at a high dose $\left(3 \times 10^{6}\right.$ viable parasites).
The fact that $P$. falciparum kahrp- parasites are capable of generating a significant infection in humans was somewhat unexpected based on the well-characterized role of KAHRP in mediating the adherence of infected erythrocytes to the microvascular endothelium, which is an important process in promoting parasite survival by evading splenic clearance [18]. The in vivo growth observed in the current study appears to be consistent with the previously reported breakthrough infections (peak parasitemia of $0.02 \%$ ) observed in 2 of 7 Aotus monkeys challenged with multiple doses of knobless $P$. falciparum parasites (up to $1 \times 10^{7}$ parasites) [17]. The delay in and variability of timing of onset of patent parasitemia in the subjects administered the highest dose suggests some in vivo adaptation of parasites to enable escape from host clearance mechanisms. In contrast to the highly reproducible growth pattern of parasites seen in experimental infection with unattenuated $P$. falciparum 3D7 parasites [48], there appears to be significant interindividual variability in this process. Such variability would likely complicate any use of this approach for in vivo immunization. Because of the unexpected breakthrough in blood stage infection at the highest dose, we investigated for selection of phenotypic or genetic changes that had taken place to explain this observation. Genome sequencing of parasites rescued into culture at maximum parasitemia prior to antimalarial treatment confirmed that parasites had not reverted back to wildtype. Similarly, ex vivo cultured parasites retained their knob-minus phenotype, and exhibited impaired cytoadherence, particularly under flow conditions.

There was evidence that parasites switched their expression of var genes during in vivo growth. Parasites administered to subjects on day 0 expressed a single dominant group $C$ var gene. This is consistent with previous evidence that, in the absence of phenotype selection, parasites often switch to expression of a group C var gene which appear to have a slower rate of silencing than other var gene types [49]. Following growth in vivo, parasites had switched away from the expression of the

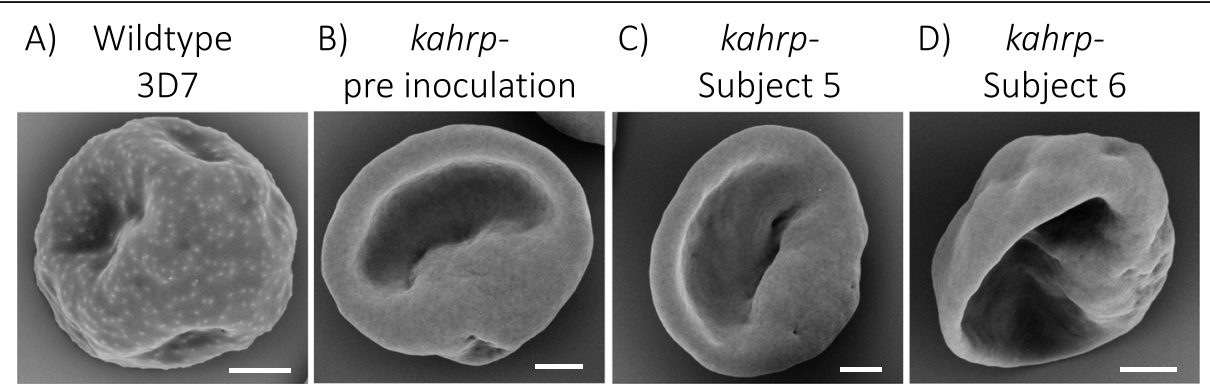

Fig. 4 Scanning electron microscopy of parasite-infected erythrocytes. P. falciparum 3D7 wild-type (A), P. falciparum 3D7 kahrp- prior to inoculation (B), P. falciparum 3D7 kahrp-cultured ex vivo from subject 5 (C) and P. falciparum 3D7 kahrp-cultured ex vivo from subject 6 (D). Scale bar $=1 \mu \mathrm{m}$ 


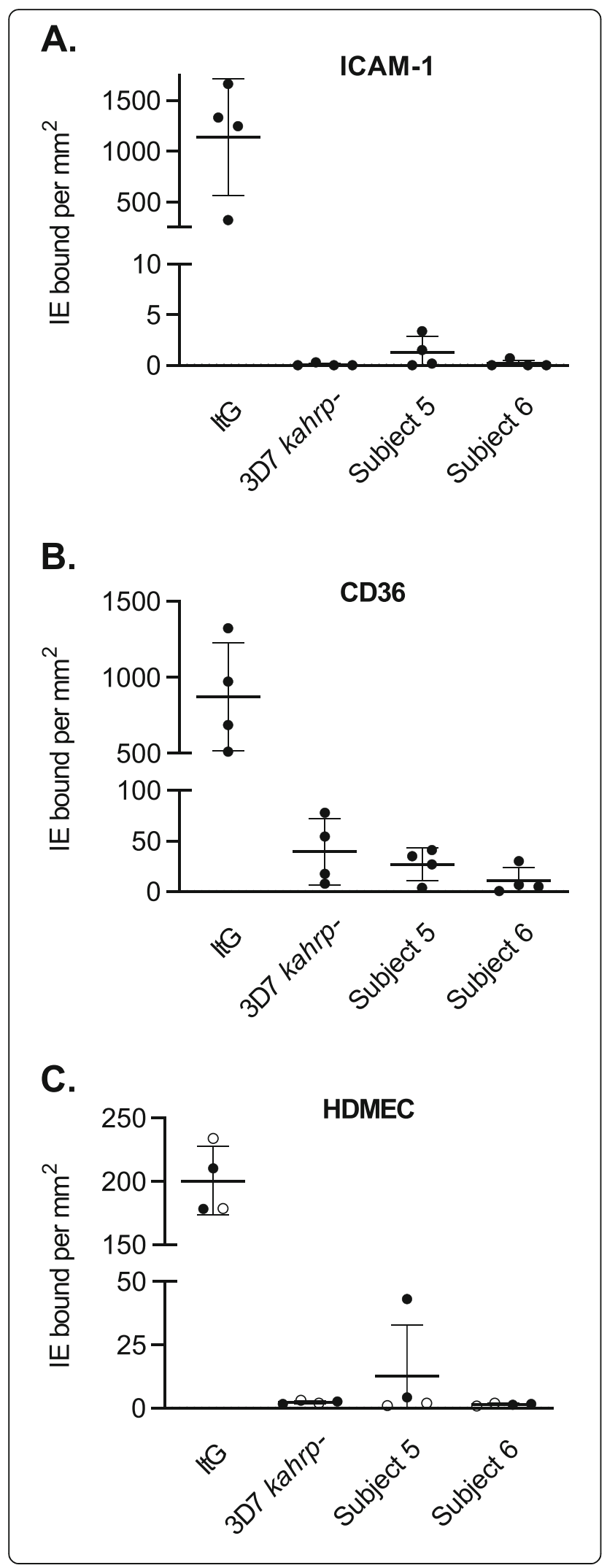

Fig. 5 Cytoadherence of parasite-infected erythrocytes. The binding of infected enythrocytes to purified ICAM-1 (A) and CD36 (B) was determined under static conditions, and binding to unstimulated (O) or TNF-stimulated (•) human dermal microvascular endothelial cells (HDMEC) was determined under flow conditions (C). Assays were performed as described in Additional File 3: Text S2. Shown are the mean \pm SD for 4 independent experiments. $P$. falciparum 3D7 kahrp- parasites cultured ex vivo from subject 5 and subject 6 were compared with parasites inoculated on day 0 . There was no difference in cytoadherence characteristics when significance was determined by unpaired $t$ test with Welch's correction $(P>0.05)$. The laboratory isolate $P$. falciparum ItG was used as a positive control. E: infected erythrocytes

single group $C$ var gene, suggesting selection in the naïve, human host, possibly for an adhesion phenotype. The increased diversity of var expression could also reflect the absence of selection for cytoadhesive variants, because the infected erythrocytes could not cytoadhere. If this were the case, then the altered environment within the host stimulated the de-repression of much of the var repertoire. Interestingly a similar observation of broad var de-repression has been made in CHMI studies using sporozoites [40]. In that case, passage through mosquitoes was inferred to have reset the epigenetic regulation of var genes, consistent with the broad, epigenetic reprogramming that occurs through sexual reproduction in higher order eukaryotes. The data we present suggests that the human host environment itself may stimulate var gene de-repression during the parasite's asexual lifecycle.

Despite the well-characterized role of knobs in the cytoadherence and sequestration of parasite-infected erythrocytes, there is some evidence that erythrocytes infected with knob-minus parasites may be capable of binding weakly to the microvascular endothelium. Knobless P. falciparum clones were found to adhere to CD36 at a level comparable to the parent 3D7 strain under static conditions, but dramatic differences were observed under flow conditions [18]. Similar results were observed in the current study, with P. falciparum kahrp- parasites exhibiting some adhesion to CD36 under static conditions (albeit at considerably lower levels than the control strain) and almost no binding under flow conditions. These results are supported by recent research using engineered human capillaries which demonstrated that erythrocytes infected with knob-minus parasites can accumulate in the post-capillary space where shear rates are lower [50]. In contrast, knob-positive parasiteinfected erythrocytes that were trypsinized to remove the surface presentation of PfEMP1 did not accumulate in vessels to any extent. Thus, we hypothesize that in the current study a limited number of P. falciparum kahrpparasites were able to bind to the microvascular endothelium in areas where shear rates were low and avoid splenic clearance. This may have been facilitated by the switch in var gene expression observed during in vivo 


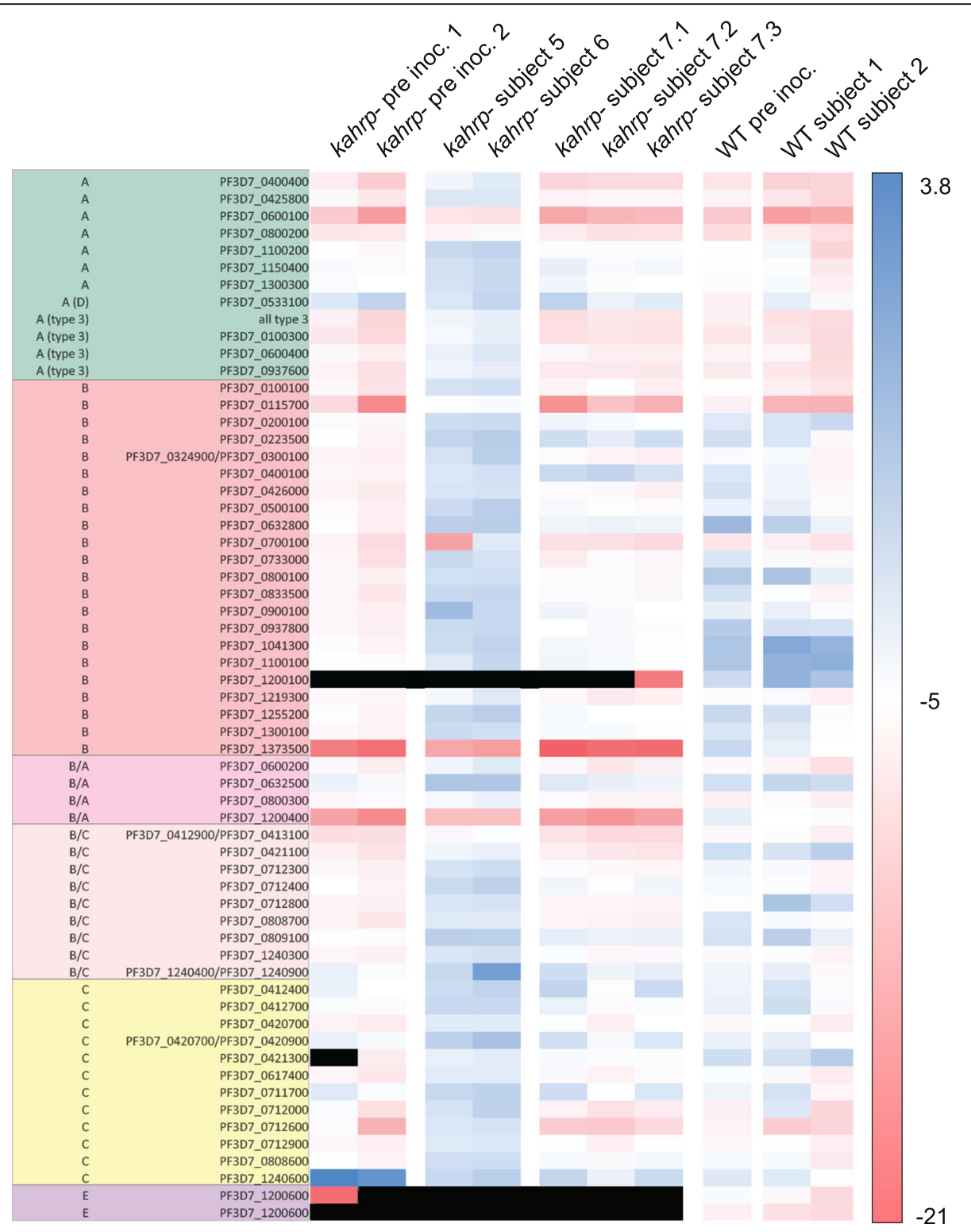

Fig. 6 Var gene expression profiles. Log fold change in var transcript copy number relative to single copies in P. falciparum 3D7 gDNA. P. falciparum 3D7 kahrp- parasites cultured ex vivo from three subjects (kahrp- subject 5, kahrp- subject 6, and kahrp- subject 7) were compared with two replicates of parasites inoculated on day 0 (kahrp- pre inoc.1 and kahrp- pre inoc.2). Parasites were collected from subjects by taking blood samples immediately prior to antimalarial treatment initiation; three replicate samples were collected from subject 7 (kahrp- subject 7.1, kahrp- subject 7.2 and kahrp- subject 7.3). The duration of in vitro culture for each sample is specified in Additional File 3: Table S1. As a control, wild-type P. falciparum 3D7 parasites cultured ex vivo from two subjects (WT subject 1 and WT subject 2) in a controlled human malaria infection study using the induced blood stage malaria model [45] were compared with parasites inoculated on day 0 (WT pre inoc.). Blue bars indicate high expression, red bars indicate low expression, and black bars indicate that no expression was detected. Rows list var gene group and accession numbers

growth to present a range of PfEMP1s on the cell surface. An alternative hypothesis is that parasites may have accumulated in the spleen and were cleared by the immune system slowly enough to maintain an infection.

Immunogenicity results indicated that the GAP vaccine was capable of eliciting a malaria-specific antibody and cell-mediated immune response when administered at the highest dose $\left(3 \times 10^{6}\right.$ viable parasites $)$.
Immunogenicity appeared to be dependent on parasite replication, evidenced by the fact that the subject administered the highest dose who did not develop parasitemia failed to generate a detectable antibody response. This finding may be consistent with evidence that killed whole parasite malaria vaccines require an adjuvant to induce robust immunity [51]. The anti-parasite humoral immune responses to the immunodominant blood-stage 
antigen MSP2 in this study were similar to those seen during natural infection against this and other bloodstage antigens, as well as when they were measured in CHMI studies using the IBSM model where subjects were infected with wild-type 3D7 parasites [52, 53]. As the preclinical primate data suggest a critical role of the spleen in the outcome of infection with knobless parasites, the effect of splenectomy on immunogenicity and clearance phenotype would require careful investigation in future clinical studies.

Although no serious safety concerns prevented completion of this stage of clinical development of the candidate vaccine, two considerations resulted in the vaccine not progressing to testing for protective efficacy in a challenge study with unattenuated parasites. Firstly, the fact that an immune response only developed in subjects who developed patent parasitemia some weeks after inoculation, and this parasitemia required termination by timed antimalarial chemotherapy, would impose significant practical limitations on the clinical development of this vaccine in the absence of other modifications to render the parasite avirulent. Secondly, two subjects administered the highest dose of GAP vaccine developed erythrocyte alloimmunization (anti-C and anti-P1 antibodies in one subject and anti-c antibodies in the other subject). This was unexpected based on our previous experience with CHMI studies using the IBSM model where alloimmunization attributed to the malaria challenge agent has not been reported previously (approximately 400 subjects in 32 studies). The fact that alloimmunization was observed in subjects who were administered a dose of parasites approximately 1000fold higher than is typically administered in CHMI studies using the IBSM model involving wild-type $P$. falciparum 3D7 $\left(3 \times 10^{6}\right.$ viable parasites vs 2800 viable parasites) suggests that the dose of parasitized erythrocytes may be a factor. The total dose of erythrocytes (including non-parasitized) administered was similar to that routinely administered (approximately $1 \times 10^{8}$ erythrocytes). We speculate that altered antigen presentation by parasitized erythrocytes with increased passage to the spleen may contribute to a higher probability of alloimmunization with the GAP vaccine. Of note, one of the 8 subjects administered a chemically attenuated blood-stage $P$. falciparum parasite vaccine in a pilot study also developed anti-c alloantibodies [10]. Although anti-P1 antibodies are not considered clinically important for transfusion reactions, anti-C and anti-c antibodies may result in a delayed transfusion reaction characterized by slow drop in hemoglobin over two weeks post-transfusion. Consultation with transfusion medicine specialists indicated there is no immediate risk if these subjects were to require emergency administration of unmatched Group O Rh (D) negative blood, and in the setting of routine blood transfusion a full cross-match would obviate such a reaction. Since both antibodies were of low titer, there is a possibility that they may diminish over time. Together, these two adverse safety findings precluded us continuing the study to test for protective immunity against virulent parasite challenge.

\section{Conclusions}

Although the results of this study overall do not support the further clinical development of the P. falciparum kahrp - strain as a blood-stage GAP malaria vaccine, they do contribute valuable new information on the pathogenicity of $P$. falciparum. Additionally, this study acts as a proof-of-concept for the use of genetically modified blood-stage malaria parasites in humans. We have previously developed IBSM models for $P$. falciparum [23], artemisinin-resistant $P$. falciparum [54], $P$. vivax [55], and $P$. malariae [56]. The use of genetically modified parasites in CHMI studies using the IBSM model offers significant opportunities in the ongoing search for interventions to reduce the burden of malaria.

\section{Abbreviations}

AE: Adverse event; CHMl: Controlled human malaria infection;

GAP: Genetically attenuated parasite; IBSM: Induced blood-stage malaria; KAHRP: Knob-associated histidine-rich protein; MSP2: Merozoite surface protein 2; PfEMP1: Plasmodium falciparum erythrocyte membrane protein 1

\section{Supplementary Information}

The online version contains supplementary material available at https://doi. org/10.1186/s12916-021-02150-x.

Additional File 1. Supplementary methods and results for subject eligibility and safety assessments. Text S1: Subject eligibility criteria. Table S1: Incidence of adverse events by subject. Table S2: Red blood cell alloantibody test results for Cohort 4 and phenotype of donor red blood cells used for GAP master cell bank manufacture.

Additional File 2. Supplementary methods and results for immunogenicity assessments of GAP vaccine. Text S1. Methodology for determining antibody mediated immune response to GAP vaccine. Text S2. Methodology for determining cell mediated immune response to GAP vaccine. Table S1. Antibodies used for flow cytometry. Figure S1. The gating strategy used to identify CD4+ T cells and subsets, as well as the expression of T cell activation markers. Figure S2. The anti-MSP2 IgM response over the course of the study in each subject. Figure S3. The IgG and IgM response to whole parasite lysate over the course of the study in each subject. Figure S4. The frequency of CD4+ T cells, Treg cells, Th1 cells and Th cells in peripheral blood mononuclear cells over the course of the study in each subject.

Additional File 3. Supplementary methods and results for in vitro assessments of GAP vaccine. Text S1. Methodology for parasite genotyping. Text S2. Methodology for cytoadherence assays. Text S3. Methodology for scanning electron microscopy. Text S4. Methodology for var gene expression analysis. Table S1. Duration of in vitro culture of P. falciparum prior to harvest for RNA extraction for var gene transcription analysis. Text S5. Results of parasite genotyping. Text S6. Results of scanning electron microscopy analysis of parasite-infected erythrocytes. Text S7. Results of cytoadherence assays. Text S8. Results 
of var gene expression analysis. Table S2. Most abundantly expressed var genes.

\section{Acknowledgements}

We thank all the volunteers who participated in the study; staff at Q-Pharm who conducted the trial; staff at the Queensland Paediatric Infectious Diseases laboratory for qPCR analysis; Dr. Dennis Shanks (Australian Defence Force Malaria and Infectious Disease Institute) for serving as an independent malaria expert; Dr James Daly (Medical Director of Pathology Services for the Australian Red Cross Lifeblood) for discussions on the red blood cell alloimmunization events; and from QIMR Berghofer Medical Research Institute we thank Rebecca Pawliw for work in developing the GAP master cell bank, and Dr. Adam Potter for manuscript preparation.

\section{Authors' contributions}

$J S M, K E$, and LS were involved in study conceptualization. JSM, RW, SS, KE, and LS were responsible for study design. RW, SS, FA, HJ, and PS were responsible for study management. JSM, AO, SW, KRT, PG, and BB conducted the study. AO, SW, AB, MWAD, JAC, DA, MJB, FDLR, CE, JH, AFC, EE, JP, AC, JS, and MFD were involved in data acquisition. MWAD, LT, JAC, DA, MJB, FDLR, $C E, J H, A F C, E M E, A C, J S, M F D$, and ATP were involved in data analysis. All authors read and approved the final manuscript.

\section{Funding}

This study was primarily funded by the National Health and Medical Research Council of Australia (NHMRC; Program Grant 1132975). The work was also supported by an Australian Research Council Grant (FL150100106), Victorian State Government Operational Infrastructure Support, and NHMRC IRIISS. JSM was supported by a NHMRC Practitioner Fellowship. The funding bodies had no role in the design of the study or collection, analysis, and interpretation of data or in writing the manuscript.

\section{Availability of data and materials}

The datasets used and/or analyzed during the current study are available from the corresponding author on reasonable request.

\section{Declarations}

\section{Ethics approval and consent to participate}

This study was approved by the QIMR Berghofer Medical Research Institute Human Research Ethics Committee (reference number: P2199), the Australian Red Cross Lifeblood Human Research Ethics Committee (reference number: 2016\#08), and the Walter and Eliza Hall Institute of Medical Research Human Research Ethics Committee (reference number: 17-05). All subjects gave written informed consent before enrollment.

\section{Consent for publication}

\section{Not applicable.}

\section{Competing interests}

KE is currently employed by GlaxoSmithKline Australia Pty Ltd. All other authors declare that they have no competing interests.

\section{Author details}

${ }^{1}$ QIMR Berghofer Medical Research Institute, Brisbane, Australia. ${ }^{2}$ Current address: PharmOut, 111 Eagle Street, Brisbane, Queensland 4000, Australia. ${ }^{3}$ Liverpool School of Tropical Medicine, Liverpool, UK. ${ }^{4}$ Centre of Defence Pathology, Royal Centre for Defence Medicine, Joint Hospital Group, Birmingham, UK. ${ }^{5}$ The University of Queensland, Brisbane, Australia. ${ }^{6}$ The Walter and Eliza Hall Institute of Medical Research, Melbourne, Australia. ${ }^{7}$ Department of Microbiology and Immunology, University of Melbourne, Melbourne, Australia. ${ }^{8}$ Department of Medical Biology, University of Melbourne, Melbourne, Australia. ${ }^{9}$ Current address: Medicines Development for Global Health Limited, 18 Kavanagh Street, Southbank, Victoria 3006, Australia. ${ }^{10}$ Bio21 Molecular Science and Biotechnology Institute, Melbourne, Australia. ${ }^{11}$ Department of Biochemistry and Molecular Biology, University of Melbourne, Melbourne, Australia. ${ }^{12}$ The Peter Doherty Institute for Infection and Immunity, Melbourne, Australia. ${ }^{13}$ Department of Medicine, Royal Melbourne Hospital, Melbourne, Australia. ${ }^{14}$ Burnet Institute, Melbourne, Australia. ${ }^{15}$ Current address: GSK, 436 Johnston Street, Abbotsford, Victoria
3067, Australia. ${ }^{16}$ Australian Institute of Tropical Health and Medicine, James Cook University, Cairns, Australia. ${ }^{17}$ Department of Medicine and Infectious Diseases, Mater Hospital and Mater Research, Brisbane, Australia.

Received: 6 July 2021 Accepted: 30 September 2021

Published online: 22 November 2021

\section{References}

1. Duffy PE, Patrick GJ. Malaria vaccines since 2000: progress, priorities, products. NPJ Vaccines. 2020;5(1):48. https://doi.org/10.1038/s41541-020-01 96-3.

2. Clinical Trials Partnership RTSS. Efficacy and safety of RTS,S/AS01 malaria vaccine with or without a booster dose in infants and children in Africa: final results of a phase 3, individually randomised, controlled trial. Lancet. 2015;386(9988):31-45. https://doi.org/10.1016/S0140-6736(15)60721-8.

3. Malaria vaccine implementation programme (MVIP): World Health Organization; [updated March 2, 2020. Available from: https://www.who.int/ news-room/q-a-detail/malaria-vaccine-implementation-programme.

4. Stanisic Dl, Good MF. Whole organism blood stage vaccines against malaria. Vaccine. 2015;33(52):7469-75. https://doi.org/10.1016/j.vaccine.2015.09.057.

5. Epstein JE, Tewari K, Lyke KE, Sim BK, Billingsley PF, Laurens MB, et al. Live attenuated malaria vaccine designed to protect through hepatic CD8(+) T cell immunity. Science. 2011;334(6055):475-80. https://doi.org/10.1126/ science. 1211548

6. Kublin JG, Mikolajczak SA, Sack BK, Fishbaugher ME, Seilie A, Shelton L, et al. Complete attenuation of genetically engineered Plasmodium falciparum sporozoites in human subjects. Sci Transl Med. 2017;9:eaad9099.

7. Reuling IJ, Mendes AM, de Jong GM, Fabra-Garcia A, Nunes-Cabaco H, van Gemert GJ, et al. An open-label phase 1/2a trial of a genetically modified rodent malaria parasite for immunization against Plasmodium falciparum malaria. Sci Transl Med. 2020;12:eaay2578.

8. Roestenberg M, Walk J, van der Boor SC, Langenberg MCC, Hoogerwerf MA, Janse JJ, et al. A double-blind, placebo-controlled phase 1/2a trial of the genetically attenuated malaria vaccine PfSPZ-GA1. Sci TransI Med. 2020;12: eaaz5629.

9. Draper SJ, Sack BK, King CR, Nielsen CM, Rayner JC, Higgins MK, et al. Malaria vaccines: recent advances and new horizons. Cell Host Microbe. 2018;24(1):43-56. https://doi.org/10.1016/j.chom.2018.06.008.

10. Stanisic DI, Fink J, Mayer J, Coghill S, Gore L, Liu XQ, et al. Vaccination with chemically attenuated Plasmodium falciparum asexual blood-stage parasites induces parasite-specific cellular immune responses in malaria-naive volunteers: a pilot study. BMC Med. 2018;16(1):184. https://doi.org/10.1186/ s12916-018-1173-9.

11. Baruch DI, Rogerson SJ, Cooke BM. Asexual blood stages of malaria antigens: cytoadherence. Chem Immunol. 2002;80:144-62. https://doi.org/1 $0.1159 / 000058839$

12. Howard RJ, Gilladoga AD. Molecular studies related to the pathogenesis of cerebral malaria. Blood. 1989;74(8):2603-18. https://doi.org/10.1182/blood. V74.8.2603.2603

13. Rowe JA, Moulds JM, Newbold Cl, Miller LH. P. falciparum rosetting mediated by a parasite-variant erythrocyte membrane protein and complement-receptor 1. Nature. 1997;388:292-5.

14. Gonzales SJ, Reyes RA, Braddom AE, Batugedara G, Bol S, Bunnik EM. Naturally acquired humoral immunity against Plasmodium falciparum malaria. Front Immunol. 2020;11:594653. https://doi.org/10.3389/fimmu.2020. 594653

15. D'Ombrain MC, Voss TS, Maier AG, Pearce JA, Hansen DS, Cowman AF, et al. Plasmodium falciparum erythrocyte membrane protein-1 specifically suppresses early production of host interferon-gamma. Cell Host Microbe. 2007;2(2):130-8. https://doi.org/10.1016/j.chom.2007.06.012.

16. Su XZ, Heatwole VM, Wertheimer SP, Guinet F, Herrfeldt JA, Peterson DS, et al. The large diverse gene family var encodes proteins involved in cytoadherence and antigenic variation of Plasmodium falciparum-infected erythrocytes. Cell. 1995;82(1):89-100. https://doi.org/10.1016/0092-8674 (95)90055-1.

17. Langreth SG, Peterson E. Pathogenicity, stability, and immunogenicity of a knobless clone of Plasmodium falciparum in Colombian owl monkeys. Infect Immun. 1985;47(3):760-6. https://doi.org/10.1128/iai.47.3.760-766.1985.

18. Crabb BS, Cooke BM, Reeder JC, Waller RF, Caruana SR, Davern KM, et al. Targeted gene disruption shows that knobs enable malaria-infected red 
cells to cytoadhere under physiological shear stress. Cell. 1997;89(2):287-96. https://doi.org/10.1016/50092-8674(00)80207-X.

19. Pawliw R, Farrow R, Sekuloski S, Jennings H, Healer J, Phuong T, et al. A bioreactor system for the manufacture of a genetically modified Plasmodium falciparum blood stage malaria cell bank for use in a clinical trial. Malar J. 2018;17(1):283. https://doi.org/10.1186/s12936-018-2435-x.

20. Rockett RJ, Tozer SJ, Peatey C, Bialasiewicz S, Whiley DM, Nissen MD, et al. A real-time, quantitative PCR method using hydrolysis probes for the monitoring of Plasmodium falciparum load in experimentally infected human volunteers. Malar J. 2011;10(1):48. https://doi.org/10.1186/1475-2 875-10-48.

21. McCarthy JS, Lotharius J, Ruckle T, Chalon S, Phillips MA, Elliott S, et al. Safety, tolerability, pharmacokinetics, and activity of the novel long-acting antimalarial DSM265: a two-part first-in-human phase 1a/1b randomised study. Lancet Infect Dis. 2017;17(6):626-35. https://doi.org/10.1016/S1473-3 099(17)30171-8.

22. McCarthy JS, Ruckle T, Elliott SL, Ballard E, Collins KA, Marquart L, et al. A single-dose combination study with the experimental antimalarials artefenomel and DSM265 to determine safety and antimalarial activity against blood-stage Plasmodium falciparum in healthy volunteers. Antimicrob Agents Chemother. 2019;64(1):e01371-19. https://doi.org/10.112 8/AAC.01371-19.

23. McCarthy JS, Sekuloski S, Griffin PM, Elliott S, Douglas N, Peatey C, et al. A pilot randomised trial of induced blood-stage Plasmodium falciparum infections in healthy volunteers for testing efficacy of new antimalarial drugs. Plos One. 2011;6(8):e21914. https://doi.org/10.1371/journal.pone.0021914.

24. Warrell DA, Gilles. HM, editors. Essential Malariology. Fourth ed: CRC Press; 2017.

25. Collins KA, Ruckle T, Elliott S, Marquart L, Ballard E, Chalon S, et al. DSM265 at 400 milligrams clears asexual stage parasites but not mature gametocytes from the blood of healthy subjects experimentally infected with Plasmodium falciparum. Antimicrob Agents Chemother. 2019;63(4):e0183718. https://doi.org/10.1128/AAC.01837-18.

26. Common Terminology Criteria for Adverse Events (CTCAE) Version 4.03. Bethesda: US Department of Health and Human Services; 2010.

27. Boyle MJ, Chan JA, Handayuni I, Reiling L, Feng G, Hilton A, et al. IgM in human immunity to Plasmodium falciparum malaria. Sci Adv. 2019;5: eaax4489.

28. Burel JG, Apte SH, Groves PL, Boyle MJ, Langer C, Beeson JG, et al. Dichotomous miR expression and immune responses following primary blood-stage malaria. JCl Insight. 2017;2(15):e93434. https://doi.org/10.1172/ jci.insight.93434.

29. Montes de Oca M, Kumar R, Rivera FL, Amante FH, Sheel M, Faleiro RJ, et al. Type I interferons regulate immune responses in humans with blood-stage Plasmodium falciparum infection. Cell Rep. 2016;17:399-412.

30. Ng SS, Souza-Fonseca-Guimaraes F, Rivera FL, Amante FH, Kumar R, Gao Y, et al. Rapid loss of group 1 innate lymphoid cells during blood stage Plasmodium infection. Clin Transl Immunology. 2018;7(1):e1003. https://doi. org/10.1002/cti2.1003.

31. Aurrecoechea C, Brestelli J, Brunk BP, Dommer J, Fischer S, Gajiia B, et al. PlasmoDB: a functional genomic database for malaria parasites Nucleic Acids Res. 2009;37(Database):D539-43. https://doi.org/10.1093/na r/gkn814.

32. Cameron DL, Schroder J, Penington JS, Do H, Molania R, Dobrovic A, et al. GRIDSS: sensitive and specific genomic rearrangement detection using positional de Bruijn graph assembly. Genome Res. 2017;27(12):2050-60. https://doi.org/10.1101/gr.222109.117.

33. Koboldt $D C$, Zhang $Q$, Larson $D E$, Shen $D$, McLellan MD, Lin L, et al. VarScan 2: somatic mutation and copy number alteration discovery in cancer by exome sequencing. Genome Res. 2012;22(3):568-76. https://doi.org/10.1101/ gr.129684.111.

34. Langmead B, Salzberg SL. Fast gapped-read alignment with Bowtie 2. Nat Methods. 2012;9(4):357-9. https://doi.org/10.1038/nmeth.1923.

35. Scheinin I, Sie D, Bengtsson $H$, van de Wiel MA, Olshen AB, van Thuij HF, et al. DNA copy number analysis of fresh and formalin-fixed specimens by shallow whole-genome sequencing with identification and exclusion of problematic regions in the genome assembly. Genome Res. 2014;24(12): 2022-32. https://doi.org/10.1101/gr.175141.114.

36. Wei Z, Wang W, Hu P, Lyon GJ, Hakonarson H. SNVer: a statistical tool for variant calling in analysis of pooled or individual next-generation sequencing data. Nucleic Acids Res. 2011;39(19):e132. https://doi.org/10.1 093/nar/gkr599.
37. Madkhali AM, Alkurbi MO, Szestak T, Bengtsson A, Patil PR, Wu Y, et al. An analysis of the binding characteristics of a panel of recently selected ICAM-1 binding Plasmodium falciparum patient isolates. PloS One. 2014;9(10): e111518. https://doi.org/10.1371/journal.pone.0111518.

38. Storm J, Jespersen JS, Seydel KB, Szestak T, Mbewe M, Chisala NV, et al. Cerebral malaria is associated with differential cytoadherence to brain endothelial cells. EMBO Mol Med. 2019;11(2):e9164. https://doi.org/10.15252/ emmm.201809164.

39. McHugh E, Carmo OMS, Blanch A, Looker O, Liu B, Tiash S, et al. Role of Plasmodium falciparum protein GEXP07 in Maurer's cleft morphology, knob architecture, and P. falciparum EMP1 trafficking. mBio. 2020;11:e03320-19.

40. Bachmann A, Petter M, Krumkamp R, Esen M, Held J, Scholz JA, et al. Mosquito passage dramatically changes var gene expression in controlled human Plasmodium falciparum infections. PLoS Pathog. 2016;12(4): e1005538. https://doi.org/10.1371/journal.ppat.1005538.

41. Duffy MF, Byrne TJ, Carret C, Ivens A, Brown GV. Ectopic recombination of a malaria var gene during mitosis associated with an altered var switch rate. Mol Biol. 2009;389(3):453-69. https://doi.org/10.1016/j.jmb.2009.04.032.

42. Duffy MF, Caragounis A, Noviyanti R, Kyriacou HM, Choong EK, Boysen K, et al. Transcribed var genes associated with placental malaria in Malawian women. Infect Immun. 2006;74(8):4875-83. https://doi.org/10.1128/IAl.01 978-05.

43. Tonkin-Hill GQ, Trianty L, Noviyanti R, Nguyen HHT, Sebayang BF, Lampah DA, et al. The Plasmodium falciparum transcriptome in severe malaria reveals altered expression of genes involved in important processes including surface antigen-encoding var genes. PLoS Biol. 2018;16(3): e2004328. https://doi.org/10.1371/journal.pbio.2004328.

44. Robinson BA, Welch TL, Smith JD. Widespread functional specialization of Plasmodium falciparum erythrocyte membrane protein 1 family members to bind CD36 analysed across a parasite genome. Mol Microbiol. 2003;47(5): 1265-78. https://doi.org/10.1046/j.1365-2958.2003.03378.X.

45. Woolley S, Fernandez M, Rebelo M, Llewellyn S, Marquart L, Mc Carthy JS. Development and evaluation of a new Plasmodium falciparum 3D7 blood stage malaria cell bank for use in malaria volunteer infection studies. Malar J. 2021;20(1):93. https://doi.org/10.1186/s12936-021-03627-z.

46. McCarthy JS. Baker M, O'Rourke P, Marquart L, Griffin P, Hooft van Huijsduijnen R, et al. Efficacy of OZ439 (artefenomel) against early Plasmodium falciparum blood-stage malaria infection in healthy volunteers. J Antimicrob Chemother. 2016;71(9):2620-7. https://doi.org/10.1093/jac/ dkw174.

47. McCarthy JS, Donini C, Chalon S, Woodford J, Marquart L, Collins KA, et al. A phase 1, placebo controlled, randomised, single ascending dose study and a volunteer infection study to characterize the safety, pharmacokinetics and antimalarial activity of the Plasmodium phosphatidylinositol 4-kinase inhibitor MMV390048. Clin Infect Dis. 2020;ciaa368.

48. Wockner LF, Hoffmann I, Webb L, Mordmuller B, Murphy SC, Kublin JG, et al. Growth Rate of Plasmodium falciparum: Analysis of Parasite Growth Data From Malaria Volunteer Infection Studies. J Infect Dis. 2020;221(6):963-72. https://doi.org/10.1093/infdis/iiz557.

49. Frank M, Dzikowski R, Amulic B, Deitsch K. Variable switching rates of malaria virulence genes are associated with chromosomal position. Mol Microbiol. 2007;64(6):1486-98. https://doi.org/10.1111/j.1365-2958.2007.05736.x.

50. Arakawa C, Gunnarsson C, Howard C, Bernabeu M, Phong K, Yang E, et al. Biophysical and biomolecular interactions of malaria-infected erythrocytes in engineered human capillaries. Sci Adv. 2020;6:eaay7243.

51. McCarthy JS, Good MF. Whole parasite blood stage malaria vaccines: a convergence of evidence. Hum Vaccin. 2010;6(1):114-23. https://doi.org/1 $0.4161 /$ hv 6.1 .10394

52. al Yaman F, Genton B, Anders R, Taraika J, Ginny M, Mellor S, et al. Assessment of the role of the humoral response to Plasmodium falciparum MSP2 compared to RESA and SPf66 in protecting Papua New Guinean children from clinical malaria. Parasite Immunol. 1995;17:493-501.

53. Kumar R, Loughland JR, Ng SS, Boyle MJ, Engwerda CR. The regulation of CD4(+) T cells during malaria. Immunol Rev. 2020;293(1):70-87. https://doi. org/10.1111/imr.12804

54. Watts RE, Odedra A, Marquart L, Webb L, Abd-Rahman AN, Cascales L, et al. Safety and parasite clearance of artemisinin-resistant Plasmodium falciparum infection: a pilot and a randomised volunteer infection study in Australia. PLoS Med. 2020;17(8):e1003203. https://doi.org/10.1371/journal.pmed.1003203.

55. Collins KA, Wang CY, Adams M, Mitchell H, Robinson GJ, Rampton M, et al. A Plasmodium vivax experimental human infection model for evaluating 
efficacy of interventions. J Clin Invest. 2020;130(6):2920-7. https://doi.org/1 $0.1172 / \mathrm{JCl} 134923$

56. Woodford J, Collins KA, Odedra A, Wang C, Jang IK, Domingo GJ, et al. An experimental human blood-stage model for studying Plasmodium malariae infection. J Infect Dis. 2020;221(6):948-55. https://doi.org/10.1 093/infdis/jiz102.

\section{Publisher's Note}

Springer Nature remains neutral with regard to jurisdictional claims in published maps and institutional affiliations.

- fast, convenient online submission

- thorough peer review by experienced researchers in your field

- rapid publication on acceptance

- support for research data, including large and complex data types

- gold Open Access which fosters wider collaboration and increased citations

- maximum visibility for your research: over $100 \mathrm{M}$ website views per year

At $\mathrm{BMC}$, research is always in progress. 